\title{
The Effect of CD 90 Expression after Intrasclera Limbal Mesenchymal Stem Cell Injection in Form Deprivation Myopia Animal Model
}

\author{
Denisa Rosati ${ }^{\mathrm{a}^{*}}$, Gatut Suhendro ${ }^{\mathrm{a}}$, Ria Sandy Deneska ${ }^{\mathrm{a}}$, Dyah Fauziah ${ }^{\mathrm{b}}$, Muhammad Rizqy \\ Abdullah $^{\mathrm{a}}$ \\ Official email address: denisarosati88@gmail.com
}

${ }^{a}$ Department of Ophthalmology, Faculty of Medicine, Universitas Airlangga, Dr. Soetomo General Hospital Surabaya, 60132, Indonesia

${ }^{\mathrm{b}}$ Department of Anatomic Pathology, Faculty of Medicine, Universitas Airlangga, Dr. Soetomo General Hospital, Surabaya, 60132, Indonesia

\begin{abstract}
Background: To investigate the capability of limbal mesenchymal stem cells (LMSCs) in increasing CD 90 expression as marker that become new fibroblast to potentially reduce myopia development in form deprivation myopia animal model.

Method: This study using cultured LMSC from rabbit (Oryctolagus cuniculus) and isolated using modification of semienzimatic methods. 24 animal models were separated into three groups, consists of control group, form deprivation myopia group (FDM), and form deprivation myopia group with LMSCs injection (FDM+LMSCs). Myopia model was made by monocular occlusion for six weeks. LMSCs was injected intrasclera after fourth week of occlusion. Enucleation was performed in sixth week. CD 90 expression of posterior sclera were evaluated using immunohistochemistry. The results between groups were analyzed using Kruskal-Wallis and Mann-Whitney with 95\% confidence interval $(\mathrm{p}<0,05)$.

Result: After four weeks of deprivation, all eyes became more myopic. LMSCs injection was done 2,5 mm posterior from limbus and two week-evaluation showed LMSCs had migrated on posterior region of the sclera. CD 90 expression showed significant differences between control and FDM+LMSCs group also FDM and FDM+LMSCs group $(\mathrm{p}<0,05)$.
\end{abstract}

Conclusion: From the current study, we concluded that LMSCs potentially control the progression of myopia in form deprived eye animal model by raising CD 90 expression that lead to additional new scleral fibroblast cells.

Keywords: Form deprivation myopia; CD 90; sclera; limbal mesenchymal stem cell

\section{Introduction}

Myopia is a common eye disorder that increasing globally in the last decade. The main structural change is a progressive elongation of the eyeball. This would increase the risk of such abnormalities like retinal detachment and macular degeneration which can cause irreversible visual damage (Li et al, 2014; Morgan et al, 2012; Pan et al, 2012).

Biochemical conditions that contribute the most to scleral biomechanics alterations are known to be decreased scleral collagen, thinning of collagen fibers, reduced glycosaminoglycans in the sclera and changes in metabolism of the extracellular matrix (Moring et al, 2007). Fibroblasts play an important role in the structure of the sclera as extracellular matrix-producing cells $(\mathrm{Wu}, 2014)$. Based on previous studies, extracellular remodeling in myopia is caused by various growth factors such as increased Tgf- $\beta$ expression, decreased bFGF expression and decreased Bone morphogenetic protein 2 (BMP-2) expression (Li et al, 2013). 
A novel method for myopia control is implantation of mesenchymal stem cell (MSC). MSC transplanted in animal sclera model form deprivation myopia showed stem cells with positive CD 90 marker can migrate to the damaged area, assisting the wound healing process and tissue regeneration. Tsai et al in 2011 stated that the sclera also has scleral progenitor cells that are capable of multipotent differentiation including becoming new fibroblasts similar to mesenchymal stem cells with CD 90 marker examination. This study to investigate the ability of limbal mesenchymal stem cell (LMSC) in increasing CD 90 expression as marker that become new fibroblast to potentially controlling myopia in form deprived eye.

\section{Literature Review}

\subsection{Myopia}

One of the most common eye disorder in the world is myopia. The prevalence of myopia itself also increases with increasing learning needs, especially in children and adolescents. The mechanism of myopia and the prevention of its progression is not known with certainty. Structural abnormalities that are known to have a close relationship with the presence of myopia are excessive elongation of the posterior segment of the eyeball. This causes a difference between the focal length and the axial length of the eye. Based on various previous experimental studies, it was found that the progressive elongation of the eyeball was due to a significant alteration of scleral extracellular matrix (Harper and Summers, 2015).

\subsection{Scleral remodelling in myopia}

Structural alterations - In high myopia, the scleral stretchability decreases and the scleral elasticity increases, especially at the posterior pole. Since the axial length is the main factor that determines a person's refractive status, changes in the components of the extracellular matrix of the sclera due to both genetic and environmental factors can cause changes in the shape of the sclera. Several studies have defined the condition of the sclera in high myopia with thinning and a decrease in the size of the collagen fiber bundles to 60-70 $\mathrm{nm}$. McBrien et al, 2001 study on tree shrew found a significant attenuation on myopia induction. The posterior sclera is found thinner by about $20 \%$ within the first 12 days of myopia development in tree shrew and decreases slowly over 3-8 months thereafter. Biochemical alterations - Biochemical changes in myopia are mainly due to changes in the main structural components of the sclera, namely collagen and proteoglycans. Various studies have revealed that there is a destruction of type I mRNA collagen expression up to $35 \%$ in myopic eyes, while the density of collagen types III and V has no significant difference (Metlapally and Wilsoet, 2015; Harper and Summers, 2014; McBrien and Gentle, 2003). Cellular alterations - The results of laboratory investigations using radiolabeled markers on DNA production showed that decreased DNA synthesis caused cellular changes in scleral fibroblasts as regulators of the scleral remodeling process. Although DNA synthesis decreased, the net number of scleral fibroblasts did not change significantly. This indicates that the increasing number of sclera per unit dry weight is due to the loss of extracellular matrix components (McBrien and Gentle, 2003).

\subsection{90 and progenitor cells in sclera}

CD90, also known as Thy-1, is a glycosylphosphatidylinositol (GPI)-anchored glycoprotein found in mesenchymal stem cell membranes. CD90 is one of the main immunophenotypic markers of mesenchymal stem cells (Sibov et al, 2012). CD90 is also known to be an adult stem cells marker. Where in several studies it was concluded that there was a relationship between the CD90 marker with CD166 and CD44. CD90 plays a role in many signaling pathways, including regulating the activity of Rho GTPases on fibroblast cells. So 
that CD90 itself functions not only as a stem cell marker but also a regulator of various stem cell signaling (Josvay et al, 2014; Barker et al, 2004). Stem cells are also found in the scleral tissue. Research by Tsai et al, 2011 succeeded in identifying scleral stem progenitor cells (SSPC) which have high cloning properties. SSPCs revealed a higher proliferation capability compared to bone marrow mesenchymal stem cells. Over $70 \%$ of SSPCs showed positive expression on various mesenchymal cell markers such as CD90, CD44, CD73 and CD144 whereas SSPCSs were negative on hematopoetic markers such as CD117, CD11b, CD34 and CD45. The differentiation ability of SSPC towards adipogenesis, chondrogenesis and osteogenesis was compared with bone marrow mesenchymal stem cells (BMMSC).

\subsection{Stem cells potential for myopia}

Various studies explain the pathogenesis of myopia progression, including remodeling of the sclera which makes the sclera thin and weak. Myopic sclera has a tendency to have a higher elasticity due to a decrease in collagen levels. Therefore, various studies have investigated various ways improving the biomechanics of the sclera to control myopia. Some recently developed study performed a minimally invasive and safe technique to deliver a number of stem cells targeting the posterior sclera. Stem cells with the capability to become various cells like fibroblasts will lead to thickened sclera in myopia. It has also been reported that mesenchymal stem cells show homing in various tissues according to their niche (Liu et al, 2010).

\section{Material and Methods}

\subsection{Animal model}

Researchers used 24, four months old, New Zealand rabbits (Oryctolagus cuniculus) weighing 2-3 kilograms. All animals were placed in the laboratory of the Institute of Tropical Disease, Universitas Airlangga with a room temperature of 25 degrees Celsius and a light-dark cycle every 12 hours. Institutional Ethical Committee of Faculty of Veterinary Medicine, Universitas Airlangga has approved our research protocols. All procedures were done with ethical standards.

\subsection{Experimental design}

All animals were divided into 3 groups of 8 rabbits each. The groups were: control group, form deprivation myopia (FDM) group and LMSC injection group (FDM+LMSC). In the control group, neither treatment nor treatment was given. In the FDM group, the right eye was occluded using a dark occluder that was glued over the eyelids. Meanwhile in the FDM + LMSC group, the right eye was injected with $0.1 \mathrm{~mL} \mathrm{LMSC}\left(1 \times 10^{5} / 0.1\right.$ $\mathrm{mL}$ ) intrasclera at the fourth week. Previously, we also performed immunofluorescence with PKH26 labeling to ensure that the injected LMSCs migrated to the posterior sclera.

\subsection{Stem cell isolation and culturing}

In this study, LMSCs were taken from rabbit corneal limbus tissue that had been isolated and cultured using primary culture media and also modified growth media. LMSCs was prepared using semi-enzymatic isolation method. To expand the stem cells, we removed the cells in culture dish in the fifth passage. Furthermore, antibodies were given with markers CD90, CD73 and CD105 for identification of stem cell surface markers.

\subsection{Streak retinoscopy and axial length measurement}


All animals in all groups underwent streak retinoscopy examination (NEITZ, Tokyo, Japan) and axial length examination with A-scan biometry (11 MHz, AxisNano, Quantel Medical). These examinations were performed at first day, fourth and sixth week after induction of myopia. Streak retinoscopy examination was performed under cyclopegia by dripping Tropicamide Eye drops 1\% (Cendo Mydriatil) every 5 minutes every 3-4 times. This procedure was performed by 2 ophthalmologists blindly. Spherical measurement was obtained from the average of the results of the two ophthalmologists. Axial length examination was performed by previously providing topical anesthesia with proparacaine hydrochloride preparations (Pantocain, Cendo).

\subsection{Histopathological tissue preparation}

Right eyes were enucleated and primarily administered with a lethal dose of pentobarbital sodium. Excision of the posterior sclera $2 \mathrm{~mm}$ from the optic nerve head was performed. It was then fixed in $4 \%$ paraformaldehyde for 48 hours. Slides of posterior scleral tissue were made with a cut size of 5-6 $\mu \mathrm{m}$ and deparaffinized using $3 \% \mathrm{H}_{2} \mathrm{O}_{2}$ and $10 \%$ goat serum. Evaluation of CD 90 expression using CD90.1 antibody (OX-7/ Thy1.1 antiMonoclonal, Cat No.GTX76208) incubated overnight at $4^{\circ} \mathrm{C}$. CD 90 expression measured by counting fibroblast cells that expressed CD 90 antibody in posterior sclera. The results were validated by an anatomic pahologist.

\subsection{Statistical analysis}

Statistical measurement were presented in descriptive table (mean and standard deviation). Significant differences of CD 90 expression between 3 groups were analyzed using Kruskal Wallis. Comparison between each group was calculated using Mann-Whitney. Statistical analysis was performed using the SPSS 25.0 statistical software. The result was considered to be statistically significant at $\mathrm{p}<0.05$.

\section{Results}

\subsection{Stem cells migration}

The immunofluorescence examination using PKH26 showed LMSCs that injected intrasclera near the limbus migrated to posterior sclera as shown in Fig.1.

\subsection{Streak retinoscopy and axial length changes}

According to the data shown in Tables 1 and 2, the results of streak retinoscopy in the subjects of the FDM group periodically found significant differences $(p=0.001)$ and also in the FDM + LMSC group ( $p=0.001)$. In the fourth week of streak retinoscopy examination, all groups found a significant difference $(p=0.013)$, as well as at the sixth week $(\mathrm{p}=0.001)$. Axial length examination using A scan biometry also found significant differences periodically in both the control group $(p=0.021)$, the FDM group $(p=0.001)$ and the FDM+LMSC group $(\mathrm{p}=0.001)$. There was also a significant difference of the sixth week axial length $(\mathrm{p}=0,049)$. 

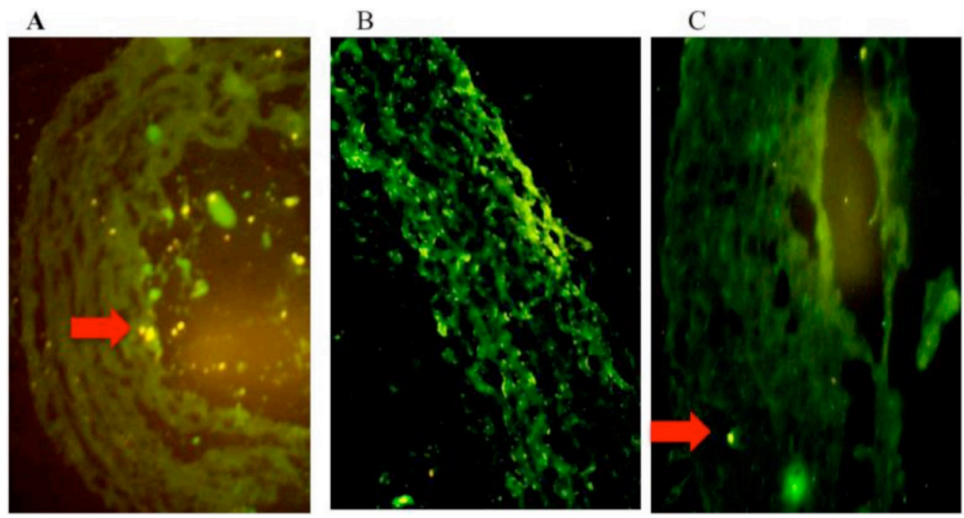

Figure 1. (A) Engravement of limbal mesenchymal stem cell in posterior sclera FDM+LMSCs group at temporal area of optic nerve (arrow); (B) Control group; (C) LMSCs at FDM+LMSCs group with inverted fluorescence microscope, 100x magnification (arrow)

Table 1. Streak retinoscopy of the subjects

\begin{tabular}{|c|c|c|c|c|c|}
\hline \multirow{2}{*}{ Group } & \multirow{2}{*}{$\mathbf{n}$} & \multicolumn{3}{|c|}{ Period } & \multirow[b]{2}{*}{$\mathbf{p}$} \\
\hline & & Week 0 & Week 4 & Week 6 & \\
\hline Control & 8 & $2,00 \pm 0,00$ & $0,88 \pm 1,36$ & $0,88 \pm 1,36$ & 0,660 \\
\hline FDM & 8 & $2,00 \pm 0,00$ & $-0,94 \pm 0,78$ & $-1,38 \pm 0,52$ & $0,001 *$ \\
\hline FDM+LMSC & 8 & $2,00 \pm 0,00$ & $0,25 \pm 0,71$ & $0,38 \pm 0,52$ & $0,001 *$ \\
\hline $\mathrm{p}$ & & 1,000 & $0,013^{*}$ & $0,001^{*}$ & \\
\hline
\end{tabular}

${ }^{\mathrm{p}} \mathrm{p}<0,05$ =significance, FDM= Form deprivation myopia, FDM+LMSC=Form deprivation myopia +limbal mesenchymal stem cell

Table 2. Axial lengths status of the subjects

\begin{tabular}{c|c|ccc|c}
\multirow{2}{*}{ Group } & \multirow{2}{*}{} & \multicolumn{3}{|c}{ Period } & p \\
\cline { 3 - 5 } & & Week 0 & Week 4 & Week 6 & \\
\hline Control & 8 & $15,30 \pm 0,10$ & $15,68 \pm 0,47$ & $15,71 \pm 0,44$ & $0,021^{*}$ \\
FDM & 8 & $15,57 \pm 0,48$ & $16,22 \pm 0,49$ & $16,33 \pm 0,54$ & $0,001^{*}$ \\
FDM+LMSC & 8 & $15,21 \pm 0,10$ & $15,90 \pm 0,50$ & $15,84 \pm 0,49$ & $0,001^{*}$ \\
p & & 0,062 & 0,066 & $0,049^{*}$ &
\end{tabular}

$* \mathrm{p}<0,05$ =significance, $\mathrm{FDM}=$ Form deprivation myopia, FDM+LMSC=Form deprivation myopia +limbal mesenchymal stem cell

\subsection{90 expression}

Statistical test with Kruskal-Wallis showed that there were differences in CD 90 expression between the three groups $(\mathrm{p}=0.00, \mathrm{p}<0.05)$. In the Mann Whitney Test to determine the differences between groups, there were significant differences between the control group and the FDM+LMSCs group $(0.12 \pm 0.35$ with 9.87 \pm 4.82 ; $\mathrm{p}=0.000, \mathrm{p}<0.05)$ and between the FDM and FDM+LMSCs groups $(0.37 \pm 0.52$ with $9.87 \pm 4.82 ; \mathrm{p}=0.000$, 
$\mathrm{p}<0.05)$. Meanwhile, the difference between the control and FDM groups was not statistically significant $(0.12 \pm 0.35$ with $0.37 \pm 0.52 ; \mathrm{p}=0.442, \mathrm{p}>0.05)$.

Table 3. Mean Expression CD 90

\begin{tabular}{|c|c|c|c|c|}
\hline \multirow[b]{2}{*}{ Group } & \multirow[b]{2}{*}{$\mathbf{n}$} & \multicolumn{3}{|c|}{ Fibroblast cells count } \\
\hline & & Mean \pm SD & Min-Max & \\
\hline Control & 8 & $0,12 \pm 0,35^{\mathrm{a}}$ & $0-1$ & \\
\hline FDM & 8 & $0,37 \pm 0,52^{\mathrm{a}}$ & $0-1$ & $0,000^{*}$ \\
\hline FDM+LMSCs & 8 & $9,87 \pm 4,82^{b}$ & $4-19$ & \\
\hline
\end{tabular}

*P significant $\alpha<0,05$

${ }^{\mathrm{ab}}$ Different superscript shows difference between two group (Mann Whitney Test)
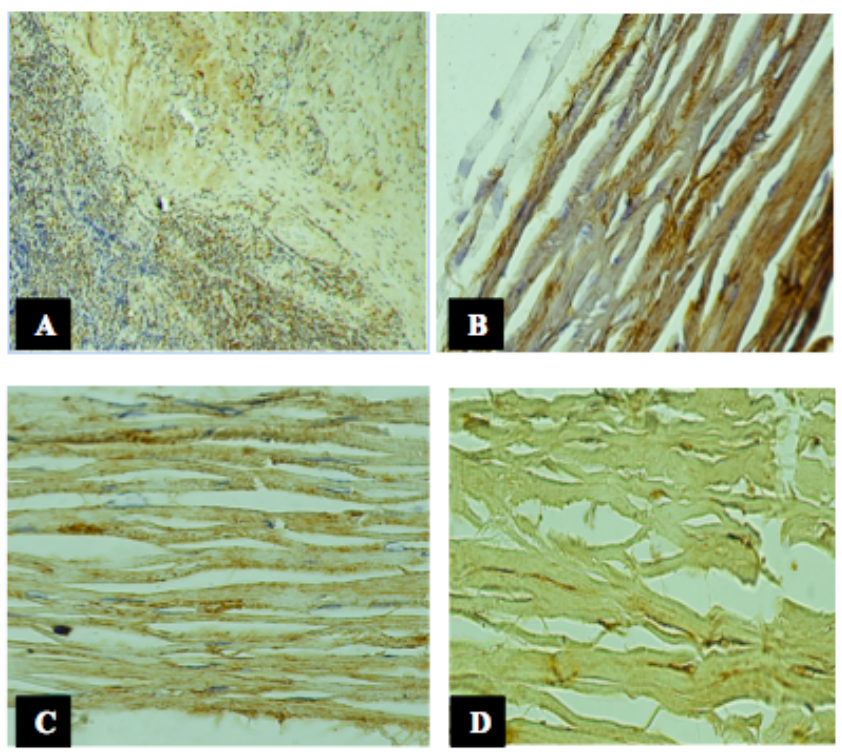

Figure 2. Immunohistochemistry staining of CD 90 expression in fibroblast scleral cells A) Control tissue; B) control group; C) form deprivation myopia group; D) form deprivation myopia+LMSCs group (diluted 1/200)

\section{Discussion}

The sclera is the outermost fibrous tissue that forms the eyeball. Sclera serves to maintain the shape of the eyeball from pressure outside and inside. The biochemical conditions of the sclera greatly determine the size and shape of the eyeball, resulting in a state of refraction. Several studies indicate that the sclera owns multipotent mesenchymal stem cells, known as scleral stem/progenitor cells (SSPCs). SSPCs have high cloning and doubling capabilities. These cells were positive on the administration of mesenchymal cell 
markers, namely CD 90, CD 105, CD 44 and negative on hematopoietic markers CD 45, CD 34 and CD 11 b. In this study, there was a significant increase in the number of fibroblast cells expressing CD 90 antibodies in the FDM group compared to the control group $(0.12 \pm 0.35$ with $9.87 \pm 4.82, p=0.011 ; p<0.05)$. and in the FDM+LMSCs group compared with the FDM group $(0.37 \pm 0.52$ with 9.87 $\pm 4.82, p=0.012 ; \mathrm{p}<0.05)$. Meanwhile, the expression of CD 90 in the control group and the FDM+LMSCs group did not show a significant difference $(0.12 \pm 1.35$ with $0.37 \pm 0.52, \mathrm{p}=0.610 ; \mathrm{p}<0.05)$.

In myopia, there is thinning of the sclera caused by a decrease in the amount of extracellular scleral matrix, especially collagen. The dominant type 1 collagen in the sclera is degraded in the myopia process. This causes a physical change in the shape of the eyeball and further affects the refractive status (Lin et al, 2008). Injection of LMSC play role in increasing fibroblast, which increase collagen synthesis resulting increases scleral thickness. This study is also based on the discovery of SSPC in the sclera based on research by Tsai et al (2011). The research was carried out by isolating the rat sclera tissue which was separated from the retinal and choroidal tissue. Then, the proliferative ability, self-repair ability and multipotent differentiation were examined using BrdU labeling, flow cytometry, RT-PCR and immunohistochemistry. It was found that there were SSPCs that could differentiate into adipogenic, chondrogenic and neurogenic strains. Chondrogenic differentiation was examined by immunohistochemical examination. It was found that SSPCs expressed chondrogenic markers just like bone marrow stem cells (BMMSCs) such as type II collagen, aggrecan and Sox9. These SSPCs were positive for mesenchymal markers CD 90, CD 44, CD 105 and CD 73. SSPCs also expressed Pax6 (a neurogenic marker and eye development), $\alpha$-SMA ( $\alpha$-smooth muscle actin and myogenic markers). SSPC that was isolated not positive for cytokeratin 12 as corneal epithelial marker in corneal epithelium, cytokeratin 19 as corneal limbal stem cell marker and also desmin as mesoderm stem cell marker in muscles. All SSPCs expressed higher type I collagen and $\alpha$-SMA than BMMSCs using immunohistochemical staining.

Our study showed that both the sclera of the control group and the sclera of the treatment (FDM) group expressed the same CD 90. This proves that in normal sclera SSPC is obtained. In the group with mesenchymal stem cell implantation (FDM+LMSCs group) there was an increase in CD 90 expression. This increase in CD 90 expression proved that stem cells in the sclera had an increase, both stem cells from injection and activation of the SSPC itself. The results of this study are consistent with a study by Kumar et al (2010) who found that mice transplanted with mesenchymal stem cells showed an increase in stem cell proliferation compared to controls with an increase in bone density. Increasing CD 90 expression leads to increasing new fibroblast cells that play role to thickened sclera. We performed intrasclera injection due to its advantage of entry the sclera-retina pathway with minimum complication such as retinal detachment or hemorrhage (Abdullah et al, 2021). On the other side, it has limited capability to expand whether we need more cells injected (Sharma et al, 1997). Whereas we did not use retrobulbar injection because it does not contribute in sclera-retina signaling loop and also potentially obstruct lymph circulation (Siegwart et al, 2002; Smith et al, 2009, Sung et al, 2020).

\section{Conclusion}

Our study reports the potential use of LMSCs for controlling myopia development. Based on this study, intrascleral injection of limbus mesenchymal stem cells in animal models of form deprivation myopia was able to increase CD 90 expression in posterior scleral animal models. The increasing CD 90 expression potentially has effect to add new fibroblast cells that play an important role in scleral remodelling in myopia control.

\section{Acknowledgements}


None.

\section{Conflict of Interest}

Nil.

\section{References}

Abdullah, M.R., Suhendro, G., Deneska, R.S., Fauziah, D., Notobroto, H.B., Lestari, A. Y., Rosati, D. 2021. Effect of Limbal Mesenchymal Stem Cell in Form Deprivation Myopia Animal Model. Annals of R.S.C.B V1. 25 : 20001-20009

Barker T.H., Hagood J.S. 2009. Getting a Grip on Thy-1 Signaling. Biochim Biophys Acta 1793 : 921-923.

Guggenheim JA, McBrien NA. 1996. Form-Deprivation Myopia Induces Activation of Scleral Matrix Metalloproteinase-2 in Tree Shrew. Invest Ophthalmol Vis Sci. 37:1380-1395

Harper, A. and Summers, J. 2015. The Dynamic Sclera: Extracellular Matrix Remodeling in Normal ocular Growth and Myopia Development. Exp Eye Res. 133: 100-111.

Josvay, K., Winter, Z., Katona, R.L., Pecze, L, Marton, A., Buhala, A. 2014. Besides Neuro-Imaging, the Thy1-YFP Mouse could Serve for Visualizing Experimental Tumours, Inflammation and WoundHealing. Sci Rep. 4 : 1-7.

Kumar, S., Nagy, T.R., Ponnazhagan, S. 2010. Therapeutic Potential of Genetically Modified Adult Stem Cells for Osteopenia. Gene Ther 17(1):105-116.

Li, H.H., Huo, L.J., Gao, Z.Y., Zhao, F. and Zeng, J.W. 2014. Regulation of Scleral Fibroblast Differentiation by Bone Morphogenetic Protein-2. Int J Ophthalmol. 7:152-156.

Lin, H.J., Wei, C.C., Chang, C.Y. 2013. Role Of Chronic Inflammation In Myopia Progression: Clinical Evidence And Experimental Validation. Biomedicine 10. 269-281.

Lin Z, Chen X, Ge J, Cui D, Wu J, Tang F, et al. 2008. Effects of Direct Intravitreal Dopamine Injection on Sclera and Retina in Form-Deprived Myopic Rabbits. J Ocul Pharmacol Ther. 24(6):543-50.

Mcbrien, N.A. 2013. Regulation Of Scleral Metabolism In Myopia And The Role Of Transforming Growth Factor-Beta. Exp Eye Res. 114:128-40.

Mcbrien, N.A. and Gentle, A. 2003. Role Of Sclera In The Developtment And Pathological Complication Of Myopia. Progress In Retinal And Eye Research. 22: 307-338.

Metlapally, R. and Wildsoet, C. 2015. Scleral Mechanism Under Ocular Growth And Myopia. Prog Mol Biol Transl Sci. 134: 241-248.

Morgan, I.G., Ohno-Matsui, K., Saw, S.M., 2012. Myopia. Lancet 379, 1739-1748.

Moring, A.G., Baker, J.R., Norton, T.T. 2007. Modulation of Glycosaminoglycan Levels in Three Shrew Sclera During Lens-Induced Myopia Development and Recovery. Invest Ophtalmol Vis Sci. 48(7):2947-2956.

Pan, C.W., Ramamurthy, D., Saw, S.M. 2012. Worldwide Prevalence and Risk Factors for Myopia. Ophthalmic Physiol. Opt. 32, 3-16.

Sharma, T., Gopal, L., Parikh, S., Shanmugam, M., Sengamedu, B., Mukesh, B. 1997. Parabulbar Anesthesia for Primary Vitreoretinal Surgery. Ophthalmology. 1997; 104:425-428

Sibov, T.T., Severino, P., Mart, L.C., Pavon, L.F., Oliveira, D.M., Tobo, P,R., Campos, A.H., Paes, A,T., Amaro, E., Gamarra, L.F., Moreira-Filho, C.A. 2012. Mesenchymal Stem Cells from Umbilical Cord Blood: Parameters for Isolation, Characterization and Adipogenic Differentiation. Ytotechnology. 64:511-521.

Siegwart, J.T., Norton, T.T. 2002. The Time Course of Changes in mRNA Levels in Tree Shrew Sclera 
During Induced Myopia and Recovery. Invest Ophthalmol Vis Sci. 43:2067-2075.

Smith $3^{\text {rd }}$ E.L., Hung, L.F., Huang, J. 2009. Relative Peripheral Hyperopic Defocus Alters Central Refractive Development in Infant Monkeys. Vis.Res. 49:2386-2392.

Sung, M.S., Ji, Y.S., Moon, H.S., Heo, H., Park, S.W. 2020. Anterior Scleral Thickness in Myopic eyes and its Association with Ocular Parameters. Ophth Res. Doi: 10.1159/000512396.

Tsai, M.S., Lee, J.L., Chang, Y.J. And Hwang, S.M. 2004. Isolation Of Human Multipotent Mesenchymal Stem Cells From Second-Trimester Amniotic Fluid Using A Novel Two-Stage Culture Protocol. Hum. Reprod. 19, 1450-1456 Crossref Pubmed.

Tsai, C.L., Wu, P.C., Shi, S. 2011. Identification of Multipotent Stem/Progenitor Cells in Murine Sclera. Invest Ophtalmol Vis Sci. 52(8) : 5481-5487.

Wu, P.C., Tsai, C.L., Gordon, G.M., Jeong, S., Itakura, T., Patel, N., Shi, S., Fini, M.E. 2015. Chondrogenesis in Scleral Stem/Progenitor Cells and Its Association with Form-Deprived Myopia in Mice. Molecular Vision; $21: 138-147$. 